\title{
Herramienta Software para Administrar el Desarrollo de Sitios Web Accesibles según Pautas WCAG 1.0
}

\author{
Juan I. Cavalieri, Pedro L. Alfonzo, Sonia I. Mariño, Maria V. Godoy \\ Facultad de Ciencias Exactas y Naturales y Agrimensura. Universidad Nacional del Nordeste. Argentina, 9 de Julio 1449 , 3400 \\ Corrientes, Argentina \\ juanicav@hotmail.com,plalfonzo@hotmail.com,simarinio@yahoo.com,mvgg2001@yahoo.com
}

\begin{abstract}
Resumen- La accesibilidad a los contenidos web, es un campo en desarrollo y representa una manera de encarar proyectos con posibilidades de crecimiento y perdurabilidad en el tiempo. Por lo expuesto es relevante que los desarrolladores de software y las empresas, elaboren sitios accesibles, por lo cual el trabajo propuesto contribuye a esta temática. Se presenta una herramienta software orientada al análisis de las pautas WCAG 1.0, a ser utilizada en etapas tempranas de desarrollo de proyectos software, siendo los resultados registrados y desplegados en distintos formatos (reportes y gráficos).
\end{abstract}

Palabras clave: Accesibilidad Web, Validadores, Desarrollo web, WCAG 1.0.

\section{INTRODUCCIÓN}

En la sociedad del conocimiento, las empresas demandan servicios que respondan de manera rápida a las peticiones de los usuarios y sean sólidos para permanecer operativo el mayor tiempo posible, a pesar de las fallas o la falta de seguridad inherente para transmitir información de alta confidencialidad: siendo mínimas las restricciones solicitadas referentes a la accesibilidad a sus contenidos.

Se coincide con [1], en que la accesibilidad a los contenidos web, es un campo en desarrollo y atendible, considerando que representa una manera de encarar proyectos con posibilidades de crecimiento y perdurabilidad en el tiempo.

Por otra parte, la AW en la República Argentina está logrando mayor difusión desde que en el mes de noviembre del año 2010 se proclamó la Ley N 26.653 [2], de Accesibilidad a la Información y se aprobó su reglamentación en el año 2013 [3].

Para verificar si un sitio web es accesible, existen herramientas automáticas disponibles en la web que evalúan y elaboran informes detallados del cumplimiento de los puntos de verificación de las Pautas de Accesibilidad al Contenido en la Web 1.0 o WCAG 1.0 [4], y las Pautas de Accesibilidad al Contenido en la Web 1.0 o WCAG 2.0 [5] propuestas por la W3C [6], como ser TAW [7], HERA [8], EXAMINATOR [9], UNICORNIO [10], Access Monitor [11], Tingtun Checkers [12], F.A.E. 2.0. [13], Wave [14], Cinthia Says [15], AChecker [16].

Las herramientas mencionadas realizan un análisis completo del sitio, pero requieren del control en forma manual del evaluador del sitio web, en aquellos puntos de verificación en que se carece de certeza del cumplimiento.

Como una superación a estos validadores web, se han desarrollado herramientas software disponible para apoyar la gestión de las normas de AW. Entre ellas se mencionan los trabajos descriptos en [17] y [18].

En [17], se presenta una herramienta de ayuda para el análisis de sitios web, permite tanto la evaluación semi- automática como la gestión de los procesos completos de evaluación. Facilita la evaluación de la accesibilidad mediante la comprobación de las pautas WCAG 1.0.

En [18], se reporta un prototipo de sistema experto basado en casos para la generación de reportes de accesibilidad de páginas web atendiendo las pautas WCAG 2.0.

Por lo expuesto, es importante que los desarrolladores de software y las empresas, elaboren sitios accesibles, por lo cual el trabajo propuesto contribuye a esta temática. Se propone una herramienta software orientada al análisis de las pautas WCAG 1.0, a ser utilizada en etapas tempranas de desarrollo de proyectos software; y de esta manera aseguraría la accesibilidad a los contenidos del sitio desarrollado, antes de su puesta en producción.

\section{METODOLOGÍA}

La metodología utilizada el desarrollo de la herramienta informática que permite gestionar la accesibilidad a los contenidos en proyectos software consistió en:

Etapa 1: Revisión, selección y estudio de herramientas para evaluar la accesibilidad a los contenidos web.

Se realizó la revisión, selección y estudio de herramientas automáticas propuestas por la W3C [6], para evaluar la accesibilidad web.

Etapa 2: Selección del validador HERA [8] la cual realiza el análisis de sitios web basándose en la normativa WCAG 1.0., permitiendo acceder a su código fuente.

Etapa 3: Desarrollo del software HADWA (Herramienta de Apoyo al Desarrollo Web Accesible)

Se procedió a la construcción del software como soporte al análisis de la WCAG 1.0 [4] de sitios web. Para el análisis de la accesibilidad, incluida en el software de gestión se basó en el código fuente de la herramienta HERA.

\section{RESULTADOS}

\section{A. Descripción de la herramienta}

El producto software se desarrolló onsiderando una secuencia de funcionamiento (Fig. 1), vinculada estrechamente con la definición exacta de funciones que desarrolla cada uno de los usuarios del sistema: i) Programador; ii) Revisor; iii) Jefe de testing y iv) Administrador.

Figura1. Esquema de funcionamiento del sistema.

Se observa en la Fig. 1 que el proceso consiste en iniciar una revisión - usuario Programador- para luego ser evaluada en profundidad por personas especializadas -usuario Revisor-, quienes realizan las verificaciones manuales que la herramienta no pueda comprobar y emiten los informes necesarios. Estos informes pasan al jefe de testing quien decide si se deben 
realizar modificaciones en el sitio o si se encuentra aprobado según las normas aplicadas.

En el caso de que la revisión no culmine de forma exitosa y requiera de modificaciones, se retroalimentarán los resultados hacia el comienzo del proceso. En este punto se deberá comenzar una nueva revisión sobre la página, atendiendo a las modificaciones y resultados provenientes del informe de revisión. Existe un sector denominado "Administración" que si bien no participa en forma activa del proceso de revisión de una página web, constituye un nexo de comunicación entre los diferentes usuarios que participan en el sistema, para supervisar el funcionamiento general de la herramienta.

Cabe destacar que al solicitar la revisión de una página web, el sistema verifica su disponibilidad ante una revisión pendiente $\mathrm{y}$, en caso de ser posible asigna en forma automática a un revisor considerando la cantidad de trabajo pendiente. De esta manera se asegura la distribución del trabajo en forma equitativa y automática entre los revisores de la organización.

Otra funcionalidad disponible es la sección de informes estadísticos. Se destaca dado que las herramientas analizadas carecen de una opción similar y se limitan a realizar una evaluación del sitio y desplegar un resultado.

El modelo de diseño que guió el desarrollo se fundamentó en la determinación de funciones específicas para asignar un experto en el proceso de evaluación pertinente. Es aconsejable que quien desarrolla y valida el sitio sean diferentes personas, para asegurar el correcto desempeño de las funcionalidades definidas en la herramienta software.

\section{B. Funcionamiento de la herramienta}

A continuación se describen los diferentes módulos que componen el sistema desarrollado y los perfiles de usuario que acceden al mismo.

- Modulo "Nueva Revisión" - perfil "Programador-

Permite enviar la revisión de una página web (Fig. 2). El programador solicitará una nueva revisión al finalizar el proceso de programación de una página.

Al pulsar el botón "Revisar" se pueden obtener 3 resultados diferentes. i) se informa que la dirección URL ingresada no es consistente o no existe (Fig. 3); ii) la página se encuentra en un proceso de evaluación aún no finalizado, por lo que se deberá esperar a que termine el proceso completo antes de ingresar nuevamente la dirección de la página (Fig. 4) y iii) se informará si el resultado fue exitoso (Fig. 5). En tal caso, el sistema evaluará la página ingresada y almacenará los datos en la base de datos. De esta manera se logra disponer de los resultados del análisis automático su análisis y empleo en cualquier momento sin depender de la herramienta para la evaluación. Así, en la siguiente sección del esquema de funcionamiento de la herramienta, el revisor tendrá que recuperar los datos almacenados para continuar con la evaluación y no tener que considerar el tiempo que implica analizar cada una de las pautas. Además, se ingresan otros datos como la fecha, programador encargado y otros datos automáticos que resulten de interés. Por último, la herramienta asigna de manera automática al revisor encargado de continuar con la evaluación. Esto lo hace tomando aquellas pendientes de cada uno de los revisores que componen el grupo de trabajo y considera con mayor posibilidad a aquel que contenga menos trabajos pendientes.

Aun cuando el usuario programador no puede realizar evaluaciones, la herramienta despliega aquellas pendientes de finalización sin la posibilidad de interactuar con ellas. Para ello, se brinda la posibilidad de contar con un filtro previo para realizar una selección más acotada. Luego se mostrarán las evaluaciones pendientes que cumplan con el criterio de selección indicado y los datos que puedan resultar relevantes para el programador, tal como se puede observar en la Fig. 6.

- Modulo "Revisar Página" - Perfil "Revisor"-.

El modulo recupera los datos almacenados, atinentes a la solicitud previa de revisión establecida en la sección anterior; el revisor deberá verificar y completar aquellas pautas WCAG 1.0 no evaluadas en forma automática. Para ello, debe seleccionar la prioridad de evaluación con la que se trabajará (Fig. 7). Esto es de gran importancia dado que permite al usuario poder continuar con cualquier grupo de prioridades sin tener que realizar el análisis en forma secuencial. Además, se presenta una breve descripción de la pauta analizada, a fin de conceptualizar lo revisado. En color verde se observan los resultados correctos, mientras que en rojo se indican los resultados que no superaron el análisis. En general las páginas no cuentan con todas las pautas implementadas, por lo que se indica en negro con la leyenda "no aplicable" cuando no corresponde la verificación de dicho punto. Por último, entre las opciones de evaluación posible, se encuentran las pautas que deben ser analizadas en forma manual. Esto se debe a que es necesario interpretar y evaluar según el juicio de un revisor especializado, algo que el sistema no puede realizar por sí solo. Esto se verá indicado en un campo de selección de color amarillo de manera que resalte y no pase desapercibido, y en él se podrá seleccionar la opción que mejor se adapte según lo evaluado. Todo lo descripto se puede visualizar en la Fig. 8, donde se observa parte del análisis de una página web. Además, se permite el ingreso de comentarios si se considera necesario. Una vez que el revisor ha finalizado la evaluación, deberá indicar el fin de dicho proceso al final de la última prioridad. Allí cambiará el campo de selección de manera que los datos sean almacenados de forma definitiva y cambien su estado actual de "Pendientes" a "Finalizados". Lo expuesto permite al programador generar una nueva evaluación sobre la página y al revisor obtener una nueva tarea.

Al igual que en la sección anterior, el revisor puede tener acceso a las evaluaciones finalizadas en forma restringida. Ingresando a la sección de "Revisiones finalizadas" podrá ver el estado actual de las evaluaciones que fueron realizadas, junto con los datos que resulten relevantes. Esto permite que pueda conocer el estado de una evaluación realizada pero no tenga acceso a la generación de informes.

- Modulo "Generar informes" - Perfil "Jefe de testing"

Este módulo ingresa al final del proceso de evaluación de una página. Brinda la información necesaria para determinar si su resultado fue exitoso o requiere modificaciones futuras. El actor denominado "Jefe de testing" es quien puede visualizar las revisiones finalizadas y generar los informes necesarios. Éstos serán remitidos en versión impresa para el análisis y toma de decisiones.

Al igual que en el caso del programador, el jefe de testing puede observar las revisiones pendientes sin tener acceso a las mismas. Lo expuesto permite llevar un control y seguimiento, se puede observarla fecha de solicitud y la última fecha de revisión, entre otros. Además, se ofrece la posibilidad de disponer de un filtro previo para una selección más acotada.

En la sección de revisiones finalizadas, un filtro de selección permite agrupar a las páginas por proyecto, seleccionar un programador, seleccionar un revisor o restringir la búsqueda a un rango de fechas posibles (Fig. 9). La Fig. 10 permite visualizar las revisiones finalizadas que cumplen con el patrón de búsqueda ingresado en el filtro, se observa que, junto 
a la información referente a la revisión, se dispone de las opciones "ver informe" y "ver estadísticas".

Seleccionando la opción "Ver informe" (Fig. 10), se despliega la interfaz de la Fig. 11, con información correspondiente a la revisión de la página seleccionada y los comentarios del revisor. Se visualiza por prioridad, de manera que la información se despliegue ordenada, facilitando la lectura. Al finalizar el informe ( $\sin$ importar la prioridad seleccionada) se puede optar por "Generar informe". Pulsando en el mismo, se pueden solicitar los datos necesarios para completar el informe: comentarios del jefe de testing y solicitud de modificaciones (Fig. 12). Una vez rellenados los campos e indicada su finalización, se muestra la versión final, la cual incluye los datos previamente ingresados y los puntos de verificación sin la posibilidad de seleccionar prioridad. Información desplegada como vista previa de impresión del informe generado. Lo expuesto se visualiza en la Fig. 13. Si el jefe de testing define como listo el informe final, debe seleccionar la opción "Descargar" (Fig. 14), versión que podrá observar un breve título informativo y los datos de las etapas previas a la descarga. Una información relevante es la adición al final del documento del detalle de los puntos que no superaron la revisión. Esto permite al programador encargado disponer de un mayor detalle de las pautas que deben modificarse o corregirse (Figuras 15 y 16).

Como se mencionó anteriormente, se ofrece la posibilidad de visualizar algunos datos estadísticos sobre la página seleccionada.

Filtradas las opciones, en la sección "Ver estadísticas", se observa la distribución de resultado en los diferentes puntos de verificación. Internamente el sistema inicializa un contador para determinar cuántos puntos finalizaron con resultado "Bien", "Mal", "No aplicable" y "No verificado". Además se muestra la duración en días de la página seleccionada desde la solicitud de revisión hasta su finalización.

Finalmente, se muestra el número de veces que la página ha ingresado al circuito funcional de revisión y la evolución de las páginas, luego de sucesivas mejoras. Como se muestra en la Fig. 17, los datos se grafican para facilitar la comprensión.

- Modulo "Administrar" - Perfil "Administrador"

Este módulo gestiona las funciones básicas de los usuarios: alta, baja y modificación, no interviene en forma activa en el proceso de evaluación de una página web. Además, se puede tener acceso al listado de revisiones pendientes y finalizadas sin la posibilidad de actuar sobre ellas. De esta manera, el "Administrador" actúa como nexo de comunicación entre los diversos usuarios y la supervisión del funcionamiento del sistema.

La opción "Listado de activos" (Fig. 18) despliega el listado de los usuarios en condición de utilizar la herramienta. Sobre el margen derecho, se dispone de la opción "Más información" para ver los datos complementarios de cada usuario. La opción "Modificar" permite cambiar o actualizar los datos del usuario.

La opción "Listado de inactivos" despliega los usuarios dados de baja (Fig. 19). Al tratarse una baja lógica, se podrá habilitarlos nuevamente para continuar utilización la herramienta.

\section{RESULTADOS}

La sociedad del conocimiento promueve el acceso abierto a los datos, la información y los códigos software a través de diversas normativas.
En lo atinente a la AW, las, leyes vigentes y el constante crecimiento de soluciones basadas en los servicios de internet demandan la necesidad de satisfacer las normas de accesibilidad WCAG 1.0 y WCAG 2.0. Siguiendo lo pautado en la normativa existen puntos de evaluación manual, es decir, que requieren la intervención de un especialista en el tema; mientras que aquellos puntos de evaluación automática son medidos por la herramienta descripta en este trabajo

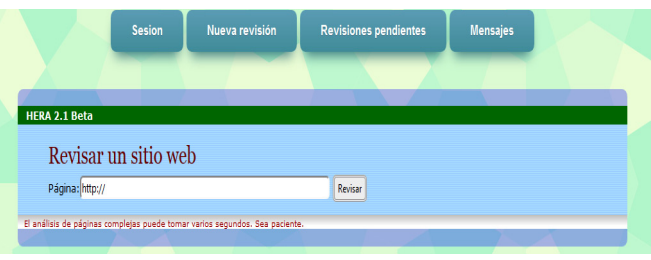

Fig. 2. Interfaz de ingreso de URL.

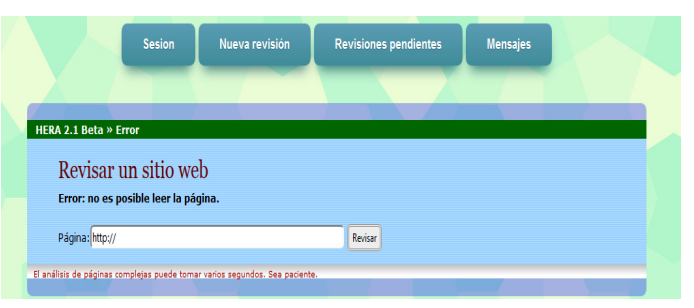

Fig. 3. Interfaz de error en URL ingresada.

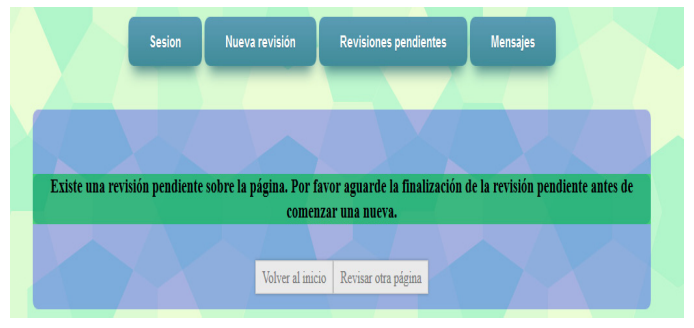

Fig. 4. 1Interfaz de revisión pendiente sobre la página ingresada.

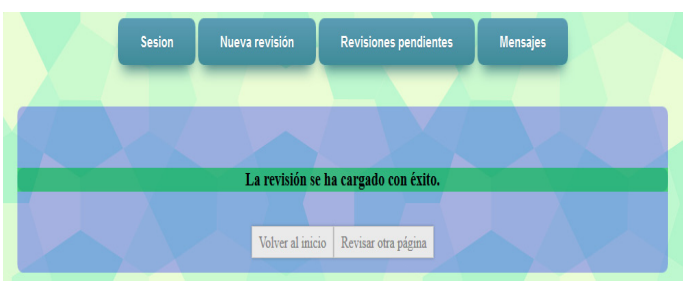

Fig. 5. Interfaz de revisión solicitada correctamente.

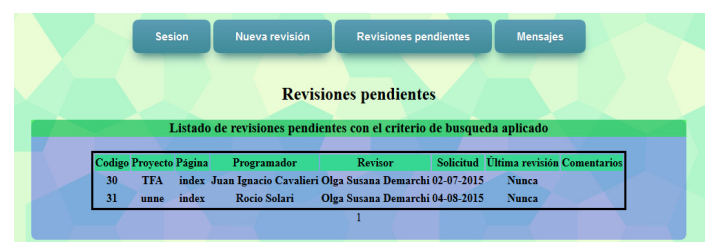

Fig. 6. Interfaz de revisiones pendientes por programador.

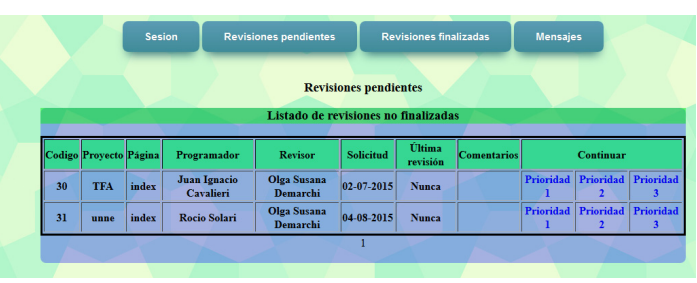

Fig. 7. Interfaz de selección de revisión pendiente a trabajar. 


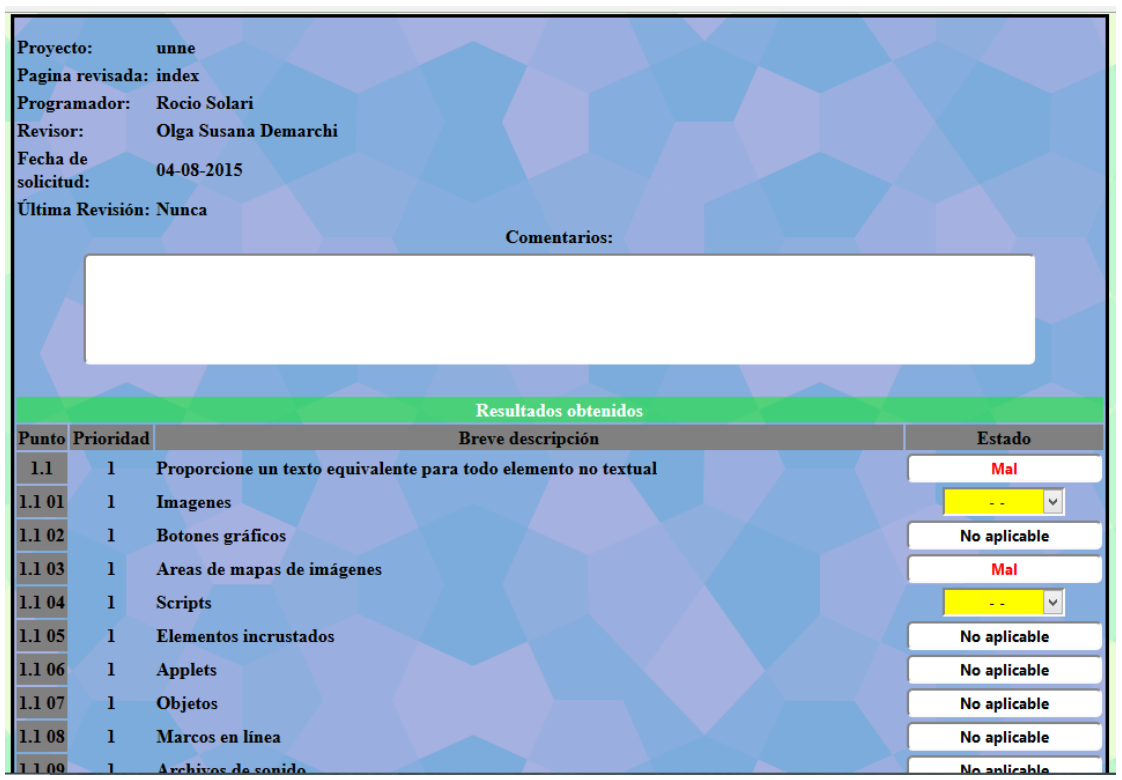

Fig. 8. Interfaz de proceso de revisión.

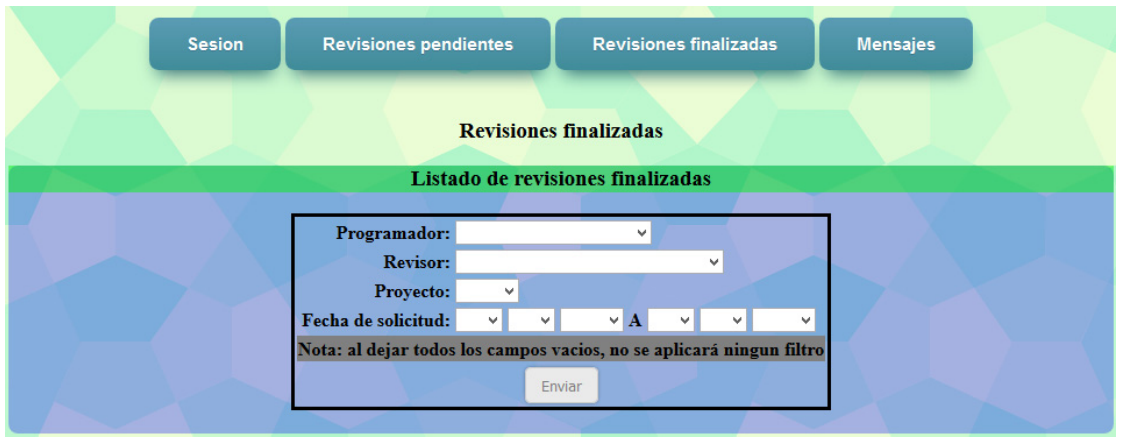

Fig. 9. Interfaz de filtro de revisiones finalizadas

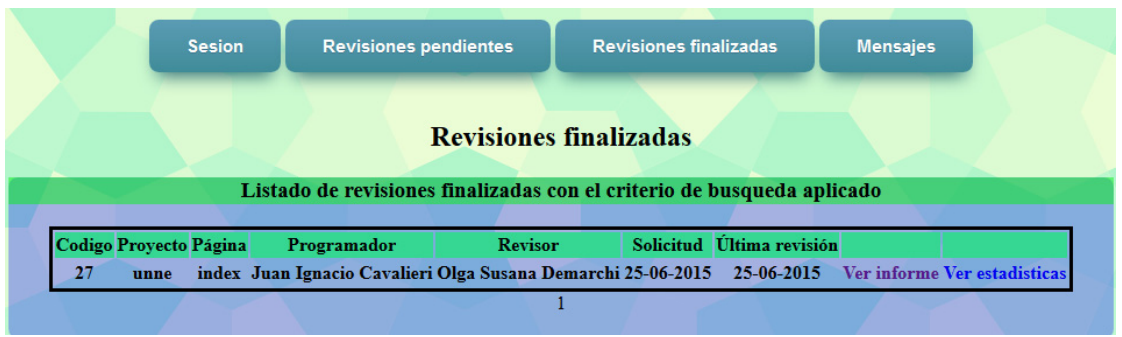

Fig. 10. Interfaz de selección de revisiones finalizadas

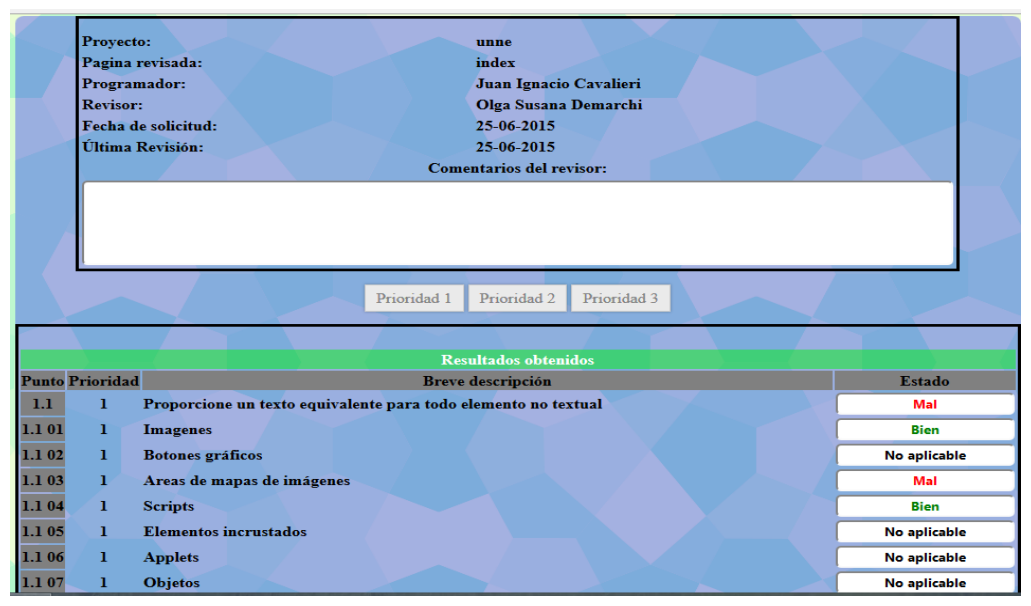

Fig. 2. Interfaz de primera etapa del informe final 


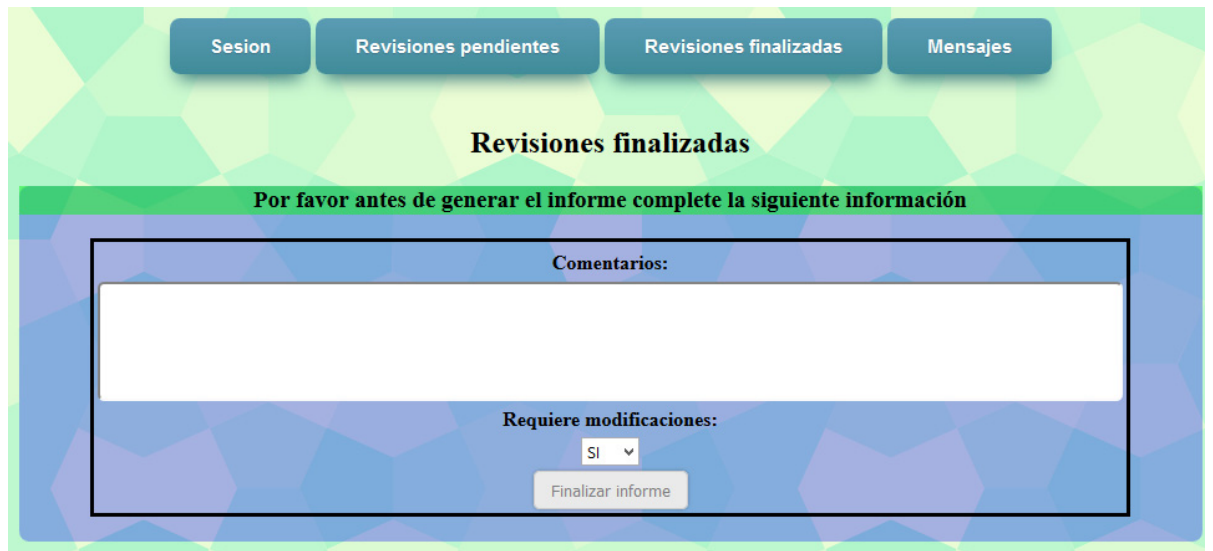

Fig. 3. Interfaz de segunda etapa del informe final

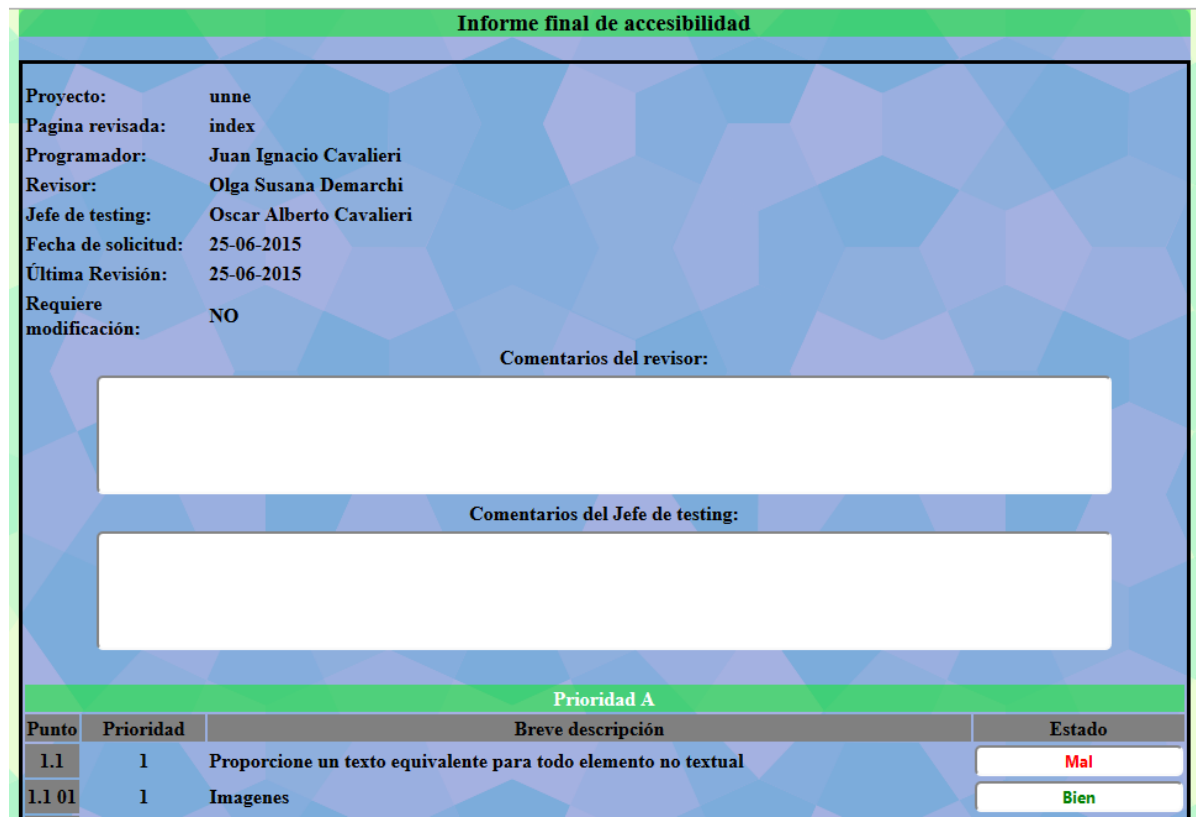

Fig. 4. Interfaz de la tercera etapa del informe final.

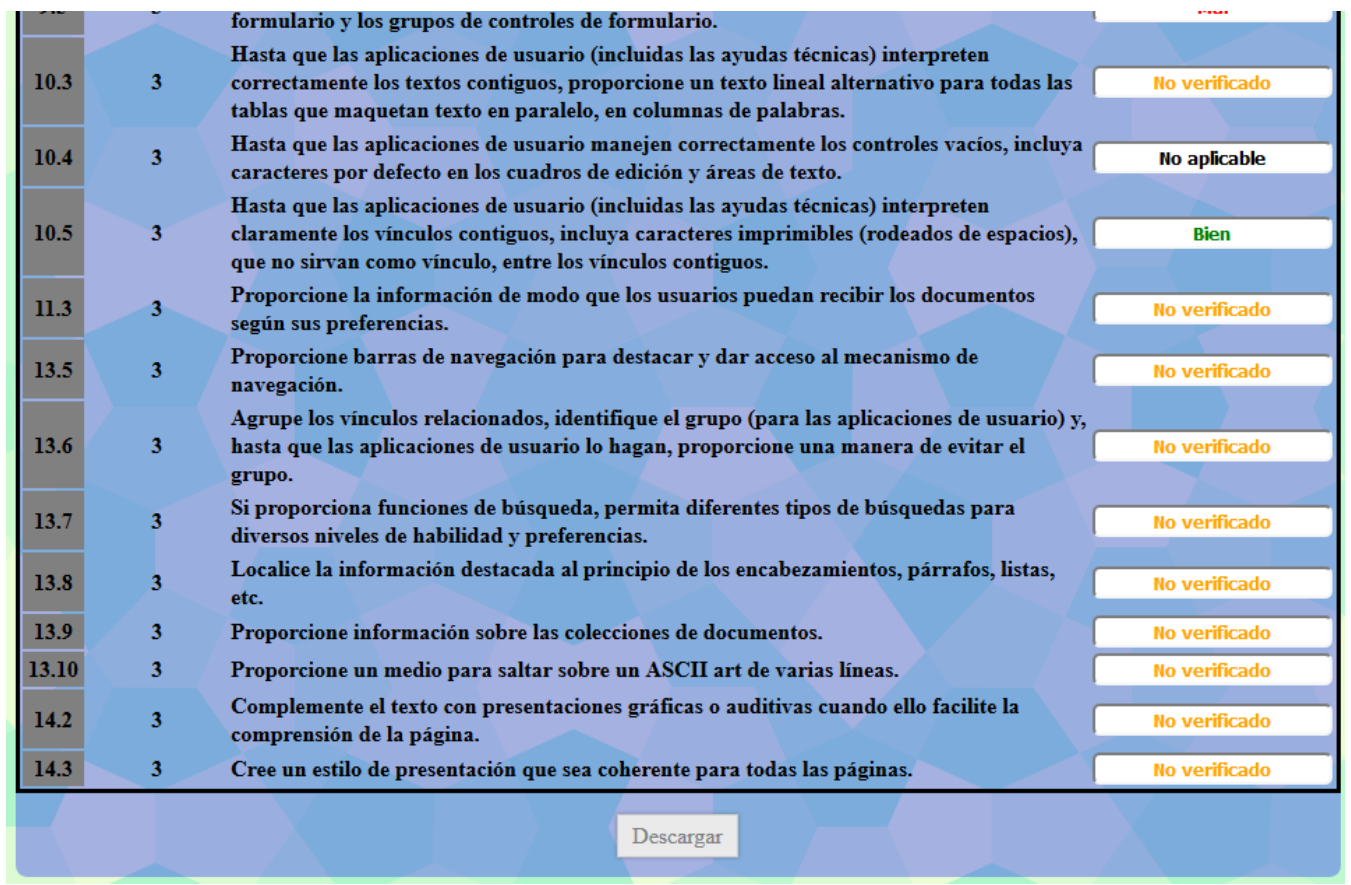

Fig. 5. Interfaz del pie de la tercera etapa del informe final. 
Informe de accesibilidad de la página index, correspondiente al proyecto denominado unne

Análisis realizado bajo la normativa WCAG 1.0

\begin{tabular}{|ll}
\hline Proyecto: & unne \\
\hline Pagina revisada: & index \\
\hline Programador: & Juan Ignacio Cavalieri \\
\hline Revisor: & Olga Susana Demarchi \\
\hline Jefe de testing: & Oscar Alberto Cavalieri \\
\hline Fecha de solicitud: & $25-06-2015$ \\
\hline Última Revisión: & $25-06-2015$ \\
\hline $\begin{array}{l}\text { Requiere } \\
\text { modificación: }\end{array}$ & NO \\
\hline
\end{tabular}

Comentarios del revisor:

\begin{tabular}{|c|c|c|c|}
\hline \multicolumn{4}{|c|}{ Comentarios del Jefe de testing: } \\
\hline \multicolumn{4}{|c|}{ Prioridad A } \\
\hline Punto & Prioridad & Breve descripción & Estado \\
\hline 1.1 & 1 & Proporcione un texto equivalente para todo elemento no textual & Mal \\
\hline 1.101 & 1 & Imágenes & Bien \\
\hline 1.102 & 1 & Botones gráficos & No aplicable \\
\hline 1.103 & 1 & Areas de mapas de imágenes & Mal \\
\hline 1.104 & 1 & Scripts & Bien \\
\hline 1.105 & 1 & Elementos incrustados & No aplicable \\
\hline 1.106 & 1 & Applets & No aplicable \\
\hline 1.107 & 1 & Objetos & No aplicable \\
\hline 1.108 & 1 & Marcos en línea & No aplicable \\
\hline 1.109 & 1 & Archivos de sonido & No aplicable \\
\hline 1.110 & 1 & Archivos multimedia & No aplicable \\
\hline 1.111 & 1 & Marcos & No aplicable \\
\hline
\end{tabular}

Fig. 6. Interfaz de parte superior del informe final descargado.

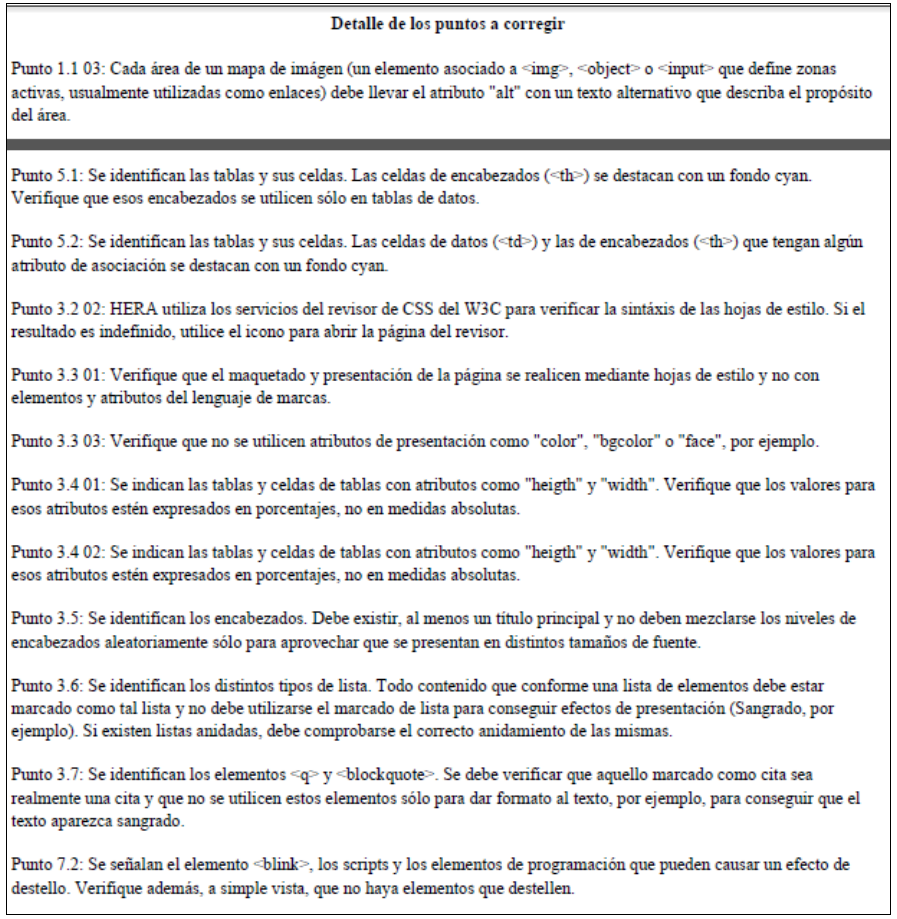

Fig. 7. Interfaz final del informe. 


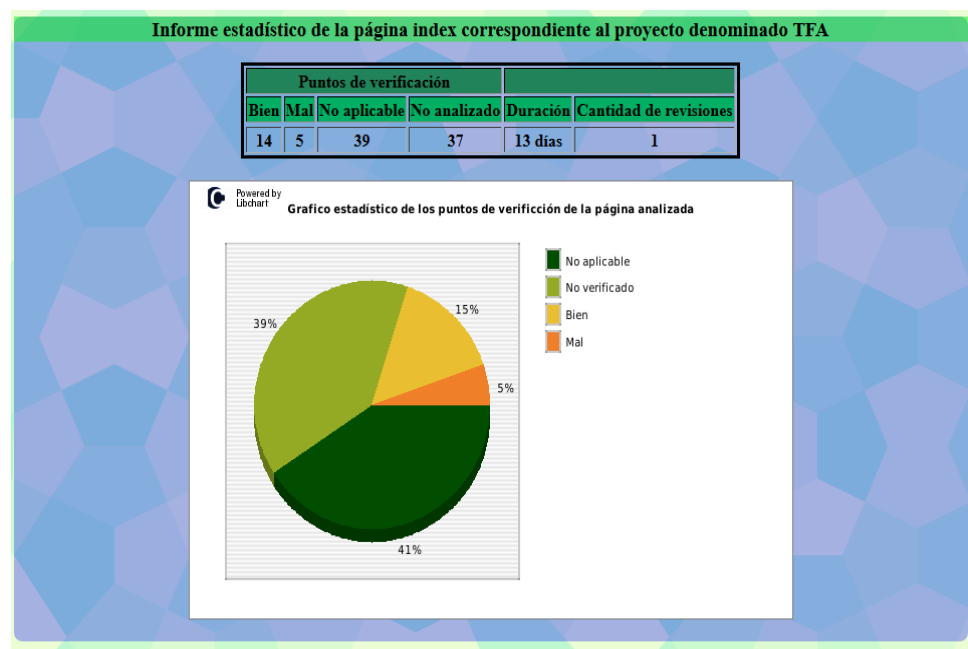

Fig. 8. Informe estadístico.

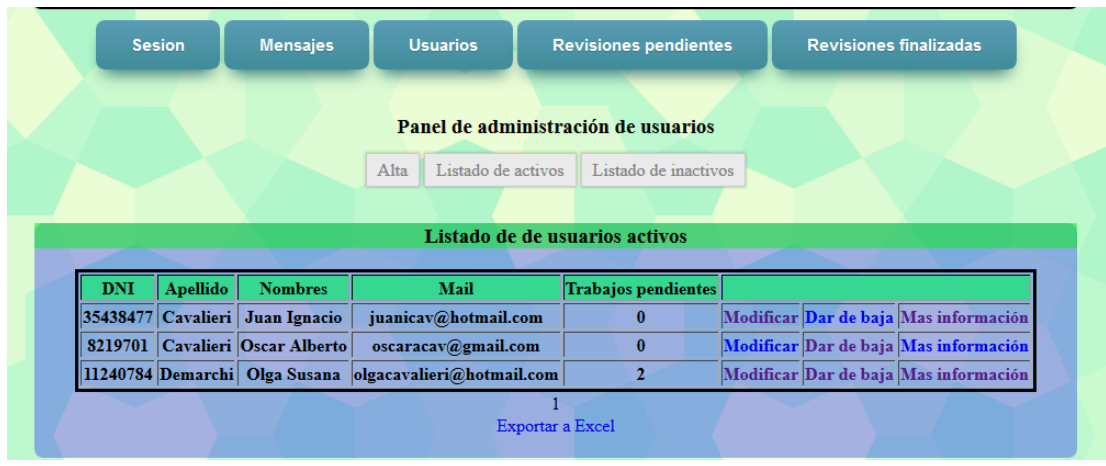

Fig. 18. Interfaz de listado de usuarios activos.

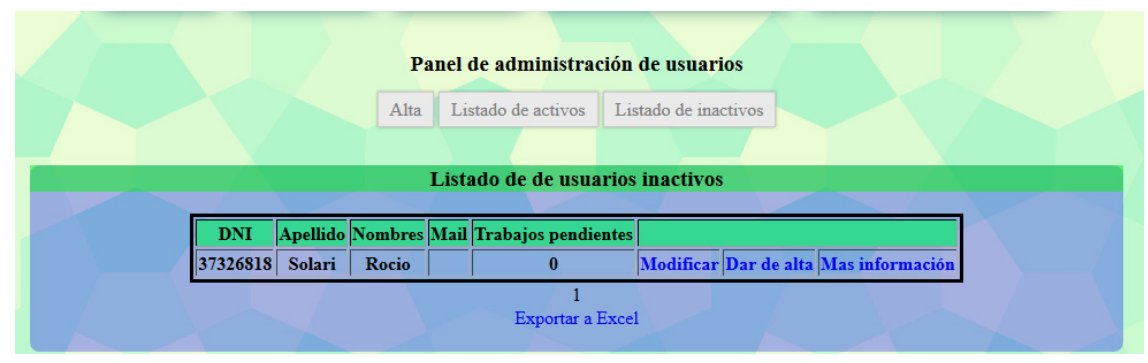

Fig. 19. Interfaz de listado de usuarios inactivos.

Se diseñó y desarrolló una herramienta para la evaluación de la AW de sitios web locales y remotos, siendo los resultados registrados y desplegados en distintos formatos (reportes y gráficos).

La propuesta se ha basado en herramienta de AW de código abierto aportando a la comunidad del software libre. Entre las ampliaciones incorporadas se destacan la interfaz de usuario que establece la secuencia de evaluación interviniendo diferentes perfiles de usuarios expertos sin necesidad de interferir entre ellos.

Se prevé a futuro incorporar opciones orientadas a brindar diversos controles, reportes y otras funcionalidades que pudieran surgir del uso.

\section{REFERENCIAS}

[1] P. L. Alfonzo, S. I. Mariño, J. I. Cavalieri y A. Gomez Codutti, Accesibilidad Web: Su abordaje en congresos argentinos de informática en el período 2012-2013. Novatica: Revista de la asociación de Técnicos de informática. 2014, no.229.

[2] INFOLEG. (2010). Aprobación de la Reglamentación de la Ley 26.653. Disponible en: < http://www.infoleg.gov.ar/infoleg
Internet/anexos/175000-179999/175694/norma.htm> [Accedido: 10 de marzo 2016].

[3] INFOLEG. (2013). Aprobación de la Reglamentación de la Ley $\mathrm{N}^{\mathrm{o}}$ 26.653. Disponible en: <http://infoleg.gov.ar/infolegInternet/ anexos/210000-214999/210143/norma.htm $>$ [Accedido: 10 de marzo 2016].

[4] WCAG (1999). Web Content Accessibility Guidelines 1.0. Disponible en: < http://www.w3.org/TR/WCAG10/ > [ Accedido: 14 de marzo de 2016].

[5] WCAG (2008). Web Content Accessibility Guidelines 2.0. Disponible en: <http://www.w3.org/TR/WCAG20>[ Accedido: 14 de marzo de 2016].

[6] W3C. Consorcio World Wide Web. Disponible en: $<$ http://www.w3c.es/> [Accedido: 10 de marzo 2016].

[7] TAW. Test de Accesibilidad Web. Disponible en: <http: // www.tawdis.net/> [ Accedido:2 de marzo 2016].

[8] HERA. Validador Automático. Disponible en: <http://www. sidar.org/hera $>$ [ Accedido: 2 de marzo 2016].

[9] EXAMINATOR. Herramienta de evaluación automática para las Pautas de Accesibilidad para el Contenido Web (WCAG) 2.0. Disponible en: <http://www.accesible.com.ar/examinator> [Accedido: 2 de marzo 2016]. 
[10] UNICORNIO Validador Unificado. Validador de código. Disponible en: <http://validator.w3.org/unicorn/?ucn_lang= es.http://www.redalyc.org/pdf/904/90426810009.pdf > [ Accedido: 2 de marzo 2016].

[11] Access Monitor. Validador automático para las normas WCAG. Disponible en: <http://www.acessibilidade.gov.pt/ accessmonitor/> [Accedido: 2 de marzo 2016].

[12] TINGTUN CHECKER. Validador automático. Disponible en: $<$ http://accessibility.tingtun.no/en/pagecheck2.0/> [Accedido: 2 de marzo 2016].

[13] FAE. Functional Accesibility Evaluator 2.0. Validador automático. Disponible en: <http://fae20.cita.illinois.edu/ $>[$ Accedido: 2 de marzo 2016].

[14] WAVE. Web Accesibility evaluation tool. Disponible en: $<$ http://wave.webaim.org/> [Accedido: 2 de marzo 2016].

[15] Cinthia Says. Validador automático. Disponible en: $<$ http://www.cynthiasays.com/Home.aspx $>$ [Accedido: 2 de marzo 2016].

[16] Checker. Open Source Web Accesibility evaluation tool. Disponible en: <http://www.atutor.ca/achecker/> [Accedido: 2 de marzo 2016].

[17] L. Mena, P. Latorre y E. Lafuente, WebA (Web Análisis): Herramienta de ayuda para el diseño y para la evaluación de la usabilidad y accesibilidad de sitios web. VII Congreso Internacional de Interacción Persona-Ordenador, 2006. Disponible en: < http://aipo.es/articulos/4/15.pdf > [Accedido: 15 de febrero de 2016].

[18] M. P. Cabrera Prieto, L. Sojos y F. Jasenia, Estudio de la normativa WCAG 2.0 y análisis de accesibilidad web para la creación de un prototipo de sistema experto basado en casos, que permita generar reportes de accesibilidad de páginas web, tomando como base un sitio web ecuatoriano. Proyecto de tesis, Ingeniería en Sistemas, Universidad Politécnica Salesiana, Ecuador, 2014. Disponible en: <http://dspace.ups.edu.ec/ handle/123456789/6290>[ Accedido: 15 de febrero de 2016].

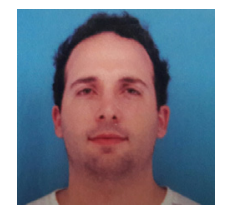

Juan I. Cavalieri. Lic. en Sistemas de Información. Graduado de la Facultad de Ciencias Exactas y Naturales y Agrimensura - Ex Becario de la Universidad Nacional del Nordeste (UNNE), Desarrollador software.

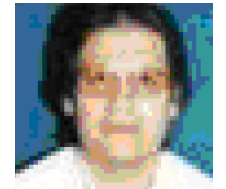

La Plata).

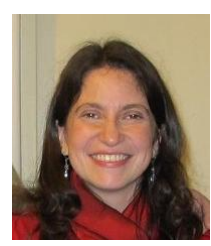

Sonia I. Mariño. Docente-Investigadora, Profesora Titular, Dedicación Exclusiva, del Departamento de Informática de la Facultad de Ciencias Exactas de la Universidad Nacional del Nordeste. Licenciada en Sistemas. Es Magíster en Informática y Computación. (UNNE - Universidad de Cantabria - España). Magíster en Epistemología y Metodología de la Investigación Científica (Facultad de Humanidades - UNNE).

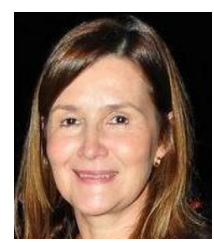

María V. Godoy. Docente-Investigadora, Profesora Titular, Dedicación Exclusiva, del Departamento de Informática de la Facultad de Ciencias Exactas de la Universidad Nacional del Nordeste. Experta en Estadística y Computación, y Licenciada en Sistemas. Es Magíster en Informática y Computación. (UNNE - Universidad de Cantabria - España). 